\title{
Effects of Water on the Stochastic Motions of Propane Confined in MCM-41- S Pores
}

\author{
Siddharth Gautam ${ }^{1 *}$, Tran Thi Bao Le ${ }^{2}$, Gernot Rother ${ }^{3}$, Niina Jalarvo ${ }^{4}$, Tingting Liu ${ }^{1}$, Eugene \\ Mamontov $^{4}$, Sheng Dai ${ }^{3}$, Zhen-An Qiao ${ }^{3}$, Alberto Striolo ${ }^{2}$ and David Cole ${ }^{1}$ \\ ${ }^{1}$ School of Earth Sciences, The Ohio State University, 125 S Oval Mall, Columbus, 43210, OH, \\ United States of America \\ ${ }^{2}$ Department of Chemical Engineering, University College London, London WC1E 6BT, United \\ Kingdom \\ ${ }^{3}$ Chemical Sciences Division, Oak Ridge National Laboratory, Oak Ridge, Tennessee 37831, \\ United States of America \\ ${ }^{4}$ Neutron Science Directorate, Oak Ridge National Laboratory, Oak Ridge, Tennessee 37831, \\ United States of America
}

*Corresponding author email: gautam.25@osu.edu

Address: School of Earth Sciences, The Ohio State University, 275 Mendenhall Laboratory, 125 S Oval Mall, Columbus, Ohio, 43210, United States of America 


\title{
Effect of Water on the Stochastic Motion of Propane Confined in MCM-41-S Pores
}

\begin{abstract}
Hydrocarbons confined in porous media find applications in a wide variety of industries and therefore their diffusive behavior is widely studied. Most of the porous media found in natural environments is laden with water, which might affect the confined hydrocarbons. To quantify the effect of hydration, we report here a combined quasielastic neutron scattering (QENS) and molecular dynamics (MD) simulation study on the dynamics of propane confined in the $1.5 \mathrm{~nm}$ wide micropores of MCM-41-S in presence of water at 230 and $250 \mathrm{~K}$. To eliminate strong incoherent signal from water and emphasize propane signal we have used heavy water $\left(\mathrm{D}_{2} \mathrm{O}\right)$. QENS data show two dynamically different populations of propane in MCM-41-S and suggest that the presence of water hinders the diffusion of propane. Weak elastic contributions to the QENS spectra suggest that only long-range translational motion of propane molecules contributes to the quasielastic broadening. MD simulations carried out using a model cylindrical silica pore of 1.6 $\mathrm{nm}$ diameter filled with water and propane agree with the experimental finding of water hindering the diffusion of propane. Further, the simulation results suggest that the slowing down of propane motions is a function of water content within the pore and is stronger at higher water contents. At high water content, the structure as well as the dynamics, both translational and rotational, of propane are severely impacted. Simulation data suggest that the rotational motion of propane molecule occurs at time scales much faster than those accessible with the QENS instrument used, and thus explains the weak elastic contribution to the QENS spectra measured in the experiments. This study shows the effects of hydration on the structure and dynamics of volatiles in porous media which are of interest for fundamental understanding and applied studies of confined fluids.
\end{abstract}




\section{Introduction}

Several industrial sectors, including catalysis and subsurface gas recovery, rely on the diffusive behavior of gases confined in nanoporous materials ${ }^{1-3}$. Of particular interest are hydrocarbons, which show very peculiar behaviors in confinement ${ }^{4-6}$. A significant amount of research effort has been devoted to the study of diffusive behavior of hydrocarbons under confinement in porous materials ${ }^{7,8}$. Both saturated ${ }^{9-14}$ and unsaturated ${ }^{15-19}$ hydrocarbons with different carbon contents and confined in various media have been studied. Among alkanes, propane offers a unique case. Because of the roughly pentagonal shape of propane molecule, this fluid exhibits the lowest melting point among alkanes ${ }^{20}$. Effects of confinement on several properties of propane have been studied by confining it in porous media differing in both pore shape as well as size ${ }^{12,13,21-25}$. While all these studies have in general found a suppression in the mobility of propane upon confinement, anomalies have been observed in the loading dependence of this suppression ${ }^{13}$. The effect of confinement on the vibrational properties of propane has also been recently documented ${ }^{26}$.

Previous work on the effects of confinement on the dynamics of propane focused on idealized systems of single-specie confinement. In natural environments, however, pores are seldom occupied by a single species. While some studies have reported the effects of presence or absence of a second species on the dynamics of one guest ${ }^{10,13,27-30}$, the most ubiquitous species that can be found in the natural pore environments - water, has largely been ignored. Although the dynamics of confined water has been studied extensively ${ }^{31,32}$, the effect of water on the dynamics of another confined species remains largely unexplored. Exceptions include Phan et al. ${ }^{33}$ and Bui et al., ${ }^{34}$ who studied the effect of water on the transport of confined methane. Recently, Le et al. reported MD simulation studies on the effect of water on the diffusion of propane in amorphous silica cylindrical pores of diameter $1.6 \mathrm{~nm}$ at $300 \mathrm{~K}^{35}$. This pore environment resembles the $1.5 \mathrm{~nm}$ wide pores in molecular sieve MCM-41-S.

To fill the gaps in our understanding of the behavior of coexisting water and a volatile, we report here a quasielastic neutron scattering (QENS) study on the effect of $\mathrm{D}_{2} \mathrm{O}$ on the dynamics of propane confined in MCM-41-S at low temperatures (230 and $250 \mathrm{~K}$ ). The results of these experiments are complemented by MD simulations, which build our previously reported simulation studies ${ }^{35}$. Both experiment and simulations suggest that water hinders the diffusion of propane in MCM-41-S pores. Further, the simulation data show that this hindering effect gets 
stronger with the water content within the pore. At the highest water content, both the structure and dynamics of the confined propane are severely restricted compared to bulk water.

In the remainder of the manuscript, we begin with detailing the experimental and simulation procedures implemented in Section 2. This is followed by defining some important quantities obtained from QENS experiments and MD simulations, and by detailing the connection between experiments and simulations in Section 3. Results from the QENS experiment are described in Section 4.1 while the structural and dynamical properties of the confined propane obtained from MD are reported in Section 4.2. In Section 5, the results from the experiments and the simulations are compared and discussed in connection with relevant literature studies. Finally, we present conclusions in Section 6. Our focus is on the properties of confined propane, while water will be treated as a medium whose principle function is to compete with propane.

\section{Experimental and Simulation Details}

2.1 Samples: The MCM-41-S sample used in the experiment was synthesized at Oak Ridge National Laboratory ${ }^{26}$. For this, Tetraethyl orthosilicate (TEOS) was added to a vigorously stirred solution of amine in ethanol and deionized water, yielding a reaction mixture of the following molar composition: 1.0 TEOS:0.27 $\mathrm{C}_{8} \mathrm{H}_{17} \mathrm{NH}_{2}: 9.09 \mathrm{EtOH}: 29.6 \mathrm{H}_{2} \mathrm{O}$. The reaction mixture was aged at ambient temperature for $18 \mathrm{~h}$ to obtain the hexagonal mesoporous silica. All ambient temperature syntheses were conducted by exposing the reaction mixture to the open atmosphere. Small amounts of deionized water were added during the aging process to compensate for the evaporation. The obtained crystalline products were recovered by filtration, washed with deionized water, and air-dried. Template removal was achieved either by calcination in air at $630{ }^{\circ} \mathrm{C}$ for $4 \mathrm{~h}$ (heating rate $2{ }^{\circ} \mathrm{C} / \mathrm{min}$ ) or by solvent extraction. The MCM-41-S sample thus synthesized had a bimodal pore distribution with pores of diameter $1.5 \mathrm{~nm}$ along with some pores of diameter 2.2 $\mathrm{nm}$. The sample surface area, determined with $\mathrm{N}_{2}$ adsorption at $77 \mathrm{~K}(\mathrm{BET})$, was $832 \mathrm{~m}^{2} / \mathrm{g}$ and its pore volume $1.2 \mathrm{~cm}^{3} / \mathrm{g}$. More details about sample characterization have been reported in an earlier publication $^{26}$.

2.2 Experiment: The Quasielastic Neutron Scattering (QENS) experiment was carried out using the backscattering instrument BASIS at the Spallation Neutron Source (SNS), Oak Ridge National 
Laboratory (ORNL), Tennessee ${ }^{36}$. This instrument provides an elastic line resolution of $3.5 \mu \mathrm{eV}$ at full width at half-maximum and an energy transfer window of $+/-120 \mu \mathrm{eV} .1 \mathrm{gm}$ of MCM-41$\mathrm{S}$ sample (1.5 nm pores) was loaded into a cylindrical aluminum cell $6 \mathrm{~mm}$ in diameter and evacuated using a vacuum pump to remove any residual proton species. Propane gas was pumped into the sample using a high-pressure syringe pump at a pressure of $1 \mathrm{bar}$, measured at $300 \mathrm{~K}$. The pressure in the sample cell was monitored using a pressure gauge on the capillary supplying the gas to it. After loading propane, the sample cell was isolated and cooled to lower temperatures. Note that cooling the sample cell resulted in a lowering of the sample cell pressure. As propane pressure corresponded to vapor densities at all times, the sample thickness was of such dimension to avoid multiple scattering. To study the effect of water on the dynamics of confined propane, another MCM-41-S sample, hydrated with $\mathrm{D}_{2} \mathrm{O}$ (10\% by weight of $\mathrm{D}_{2} \mathrm{O}$ in MCM-41-S) was used in separate QENS measurements. We note that this water loading does not quantify the amount of water that penetrated the pores. The difference between the two samples used in QENS experiments is thus mainly in terms of presence or absence of water in the pores. Measurements were taken at the temperatures $250 \mathrm{~K}, 230 \mathrm{~K}$ and $10 \mathrm{~K}$. The lowest temperature measurement was used to define the instrumental resolution. Spectra of hydrated as well as dehydrated MCM-41-S were subtracted from the respective propane loaded spectra to account for silica and water background. This subtraction also removes any contribution to the QENS spectra from the silanol groups on the pore surface. The subtracted spectra thus represent signals from propane alone. QENS data were reduced and analyzed using the software package DAVE ${ }^{37}$.

2.3 Simulations: The preparation of the simulation cell used in this work has been described elsewhere ${ }^{35}$. It consisted of two stages - preparing a cylindrical pore of amorphous silica, and then loading water and propane molecules in this pore. For the first stage, a $\beta$-cristobalite supercell was melted at $7000 \mathrm{~K}$, equilibrated in the liquid state and then quenched by cooling it at a rate of 4 $\mathrm{K} / \mathrm{ps}$ to $300 \mathrm{~K}$. A cylindrical pore of diameter $16 \AA$ was then carved out from the simulation cell by removing all atoms that lay within $8 \AA$ from the $\mathrm{X}$-axis. The resulting pore was oriented along $\mathrm{X}$-axis. Removing atoms in this manner resulted in dangling $\mathrm{Si}$ and $\mathrm{O}$ atoms. These were saturated with hydroxyl groups and hydrogen atoms, respectively. In the second stage, a desired number of water and propane molecules were placed at each side of the cylindrical pore, along the $\mathrm{X}$ direction. As simulations proceeded, water and propane spontaneously filled the pore and distributed across both pore and bulk volumes. Once equilibrium is reached, the propane density in the bulk was 
calculated from density profiles along the X-direction. The reservoir was removed after fluid molecules corresponding to the appropriate densities were adsorbed in the pore. More details on the two stages of sample preparation can be found elsewhere ${ }^{35}$.

To prepare the model MCM-41-S using the melting of a $\beta$-cristobalite crystal as outlined above, we used the Morse-type potential developed by Demirlap et. al. ${ }^{38}$ to model the interaction between $\mathrm{Si}$ and $\mathrm{O}$ atoms. Once the MCM-41-S model pore was prepared, the substrate was modeled using the $\mathrm{CLAYFF}^{39}$ force field in all subsequent simulations. Water molecules were modeled with TIP4P/Ice ${ }^{40}$ force field while TraPPE-UA ${ }^{41}$ force field was used to model propane molecules. Following the TraPPE-UA convention, all interactions were cut-off at $14 \AA$. Long-range electrostatic interactions were treated with the particle mesh Ewald (PME) ${ }^{42}$ method and LorentzBerthelot mixing rules were used to estimate the parameters for cross-terms ${ }^{43}$. Periodic boundary conditions were applied in all directions. This resulted in an infinitely long cylindrical pore along the Cartesian $\mathrm{X}$ direction.

Several different fluid compositions were simulated to systematically study the effect of water on the dynamics of confined propane. The initial number of propane molecules adsorbed in the pore was determined by GCMC simulations at 1 bar and $300 \mathrm{~K}$ to match the experimental conditions at the time of gas loading in the experiments. However, the amount of propane (5 molecules) was too small for extracting quantities with good statistics, therefore a slightly higher propane loading of 22 molecules was inserted in the simulation cell. Several water loadings were used, as summarized in Table 1. Each of the samples listed in Table 1 were simulated at 230 and $250 \mathrm{~K}$. As noted earlier, the focus of this paper is on the dynamics of propane. Quantities used for comparison between the experiments and simulations (i. e., intermediate scattering functions) were calculated for the systems Dry and Hydrated 1 (D and H1 in Table 1) at $230 \mathrm{~K}$. All simulated systems were equilibrated for 80-100 ns before a production run of $2 \mathrm{~ns}$. The production run was limited to $2 \mathrm{~ns}$ as our intention was to mainly compare the simulations with QENS experiments which probe time scales below 1 ns. The production run was repeated for up to 3 times, and no significant deviation was observed in the results obtained. 
Table 1. System composition (number of propane and water molecules) for our MD simulations in model MCM-41-S pore.

\begin{tabular}{|l|c|c|}
\hline Simulation Sample & Number of Propane molecules & Number of water molecules \\
\hline Dry (D) & 22 & 0 \\
\hline Hydrated 1 (H1) & 22 & 221 \\
\hline Hydrated 2 (H2) & 22 & 271 \\
\hline Hydrated 3 (H3) & 22 & 321 \\
\hline Hydrated 4 (H4) & 22 & 362 \\
\hline
\end{tabular}

\section{Important quantities and connection between QENS and MD simulations}

QENS and MD simulations are often used in combination to study the stochastic motion of molecules ${ }^{7}$. This is because both techniques access similar length and time scales ${ }^{7}$, although advances in computations have made the range of length and time scales accessible to MD simulations relatively larger and longer respectively. The self-diffusion coefficient $(D)$ is obtained from QENS experiments by analyzing the broadening of an elastic line due to the stochastic motions constituting self-diffusion ${ }^{44}$. From MD simulations, $D$ can be obtained from the long-time slope of mean squared displacement (MSD) vs time plots ${ }^{43}$ :

$D=\lim _{t \rightarrow \infty} \frac{\left\langle\left|\boldsymbol{r}_{\boldsymbol{i}}\left(t+t_{0}\right)-\boldsymbol{r}_{\boldsymbol{i}}\left(t_{0}\right)\right|^{2}\right\rangle}{2 n_{d} t}$

In Eq. (1), the quantity in the numerator is the MSD, with $\boldsymbol{r}_{i}\left(t+t_{0}\right)$ and $\boldsymbol{r}_{i}\left(t_{0}\right)$ being the positions of the $i^{\text {th }}$ entity (atoms or molecules) at times $t+t_{0}$ and $t_{0}$, respectively; the angular brackets denote ensemble average. The quantity $n_{d}$ in the denominator stands for the number of degrees of freedom. Typically, center of mass positions of the molecules are used for calculating $\boldsymbol{r}_{i}$.

In our previous study ${ }^{23}$, we showed that a more direct comparison can be made between the QENS experiments and MD simulations by calculating quantities that are directly related to the observed signal in the QENS experiments from the simulated trajectories. In a QENS experiment on a sample with hydrogen atoms, the observed signal is proportional to the incoherent scattering law $S_{\text {inc }}(Q, \omega)$, a quantity that encodes information on the structure and dynamics in the sample by 
virtue of its dependence on $Q$ and $\omega$, respectively*. The incoherent scattering law is a Fourier transform of the self-intermediate scattering function (ISF), $I(Q, t)$. ISF can be directly calculated from the simulated molecular trajectories using the expression

$$
I(Q, t)=\left\langle\exp \left(i \boldsymbol{Q} \cdot\left[\boldsymbol{r}_{\boldsymbol{i}}\left(t+t_{0}\right)-\boldsymbol{r}_{\boldsymbol{i}}\left(t_{0}\right)\right]\right)\right\rangle
$$

In Eq. (2), $i=\sqrt{ }-1$ and averages are carried out over all atoms/molecules and time origins $t_{0}$ and different $\boldsymbol{Q}$ with the same magnitude. The last averaging is the powder averaging necessary for comparisons to experiments using powder samples with no preferred orientation. Further, this function can be calculated for contributions from translational and rotational motions by separating the co-ordinates of an interaction site (for example $\mathrm{CH}_{3}$ ) $(\boldsymbol{r})$ into co-ordinates of the center of mass $(\mathrm{COM})$ of the molecules $\left(\boldsymbol{r}_{C O M}\right)$ and co-ordinates of that site in the center of mass frame $(\boldsymbol{d})$. Thus, $\boldsymbol{r}=\boldsymbol{r}_{C O M}+\boldsymbol{d}$

Purely translational motion of the molecules can be studied by following the evolution of $\boldsymbol{r}_{\text {СОм in }}$ time, whereas rotational motion can be studied by following the evolution of a unit vector $(\boldsymbol{e})$ along $\boldsymbol{d}$ in time. Self-intermediate scattering functions for the two motions can be calculated by replacing $\boldsymbol{r}$ in Eq. 2 by $\boldsymbol{r}_{\mathrm{COM}}$ to obtain the translational intermediate scattering function (TISF) and by $\boldsymbol{e}$ to obtain rotational intermediate scattering function (RISF).

The scattering law obtained from QENS experiments on a diffusive system is often modeled with a Lorentzian function to represent the quasielastic broadening ${ }^{44}$. The corresponding model in the inverse Fourier space for the ISF is therefore an exponential decay function, as a Lorentzian and exponential decay function form a Fourier transform pair. Thus,

$S_{\text {inc }}(Q, \omega) \sim L(\Gamma(Q), \omega) \sim \frac{\Gamma(Q)}{\left(\Gamma^{2}(Q)+\omega^{2}\right)} \quad \Leftrightarrow \quad I(Q, t) \sim e^{-t / \tau(Q)}$

\footnotetext{
${ }^{*} \boldsymbol{Q}=\boldsymbol{k}_{\boldsymbol{i}}-\boldsymbol{k}_{\boldsymbol{f}}$ is the wave-vector transfer in the event of scattering of the neutron by a sample under study, $\boldsymbol{k}_{\boldsymbol{i}}$ and $\boldsymbol{k}_{\boldsymbol{f}}$ being the wave-vector of the neutron before and after the scattering and $\hbar \omega$ is the energy transfer that occurs between the neutron and the sample on a scattering event. $\hbar$ is the reduced Planck constant.
} 
In Eq. (4), $L(\Gamma(Q), \omega)$ is a Lorentzian function of $\omega$, centered at $\omega=0$ and its half-width at halfmaximum (HWHM) is $\Gamma(Q)$. The exponential decay function $e^{-t / \tau(Q)}$ is characterized by the decay time constant $\tau(Q)$ which is related to the HWHM of the corresponding Lorentzian function as

$\Gamma(Q)=\frac{\hbar}{\tau(Q)}$

Thus, for a diffusive system, the decay constant obtained from modeling the simulated ISF with an exponential decay can be converted to a corresponding energy using Eq. (5), which is directly comparable with the HWHM of the Lorentzian used to model the experimentally measured scattering law.

Another important quantity that can be calculated from the simulated trajectories is the rotational correlation function (RCF). RCF can be used to assess the rotational motion of molecules and obtain the relevant time scales. RCF can be calculated using the following expression

$R C F=\left\langle\boldsymbol{e}_{i}\left(t+t_{0}\right) \cdot \boldsymbol{e}_{i}\left(t_{0}\right)\right\rangle$

In Eq. (6), angular brackets denote ensemble averages, and $\boldsymbol{e}_{i}$ is a unit vector rotating with molecule $i$. In the present case we use a unit vector along the $\mathrm{CH}_{3}-\mathrm{CH}_{2}$ vector of a propane molecule.

\section{Results}

\subsection{QENS experiments}

3 Figure 1 shows a comparison of the spectra obtained from propane in (a) dry and (b) hydrated 4 (with $\mathrm{D}_{2} \mathrm{O}$ ) MCM-41-S sample at 230 and $250 \mathrm{~K}$. Also shown are the spectra of a Vanadium can 5 measured at $300 \mathrm{~K}$, which serves as a measure of the instrumental resolution, and evacuated MCM-

6 41-S, also measured at $300 \mathrm{~K}$. The spectrum of evacuated MCM-41-S is almost congruent with 7 that of Vanadium, suggesting an absence of a mobile hydrogen bearing species in MCM-41-S. 8 When $\mathrm{D}_{2} \mathrm{O}$ is added to this evacuated sample and the sample cell is cooled to $10 \mathrm{~K}$, the resulting 9 spectrum (shown in (b)) is congruent with the Vanadium spectrum too, and hence can be used to 10 account for the instrument resolution. A comparison of the spectra of propane in dry and moist samples demonstrates that addition of $\mathrm{D}_{2} \mathrm{O}$ decreases the quasielastic broadening in the signal 
12 produced by the stochastic motion of propane molecules. Hence, our experiments confirm that 13 addition of $\mathrm{D}_{2} \mathrm{O}$ suppresses the motion of propane in MCM-41-S.
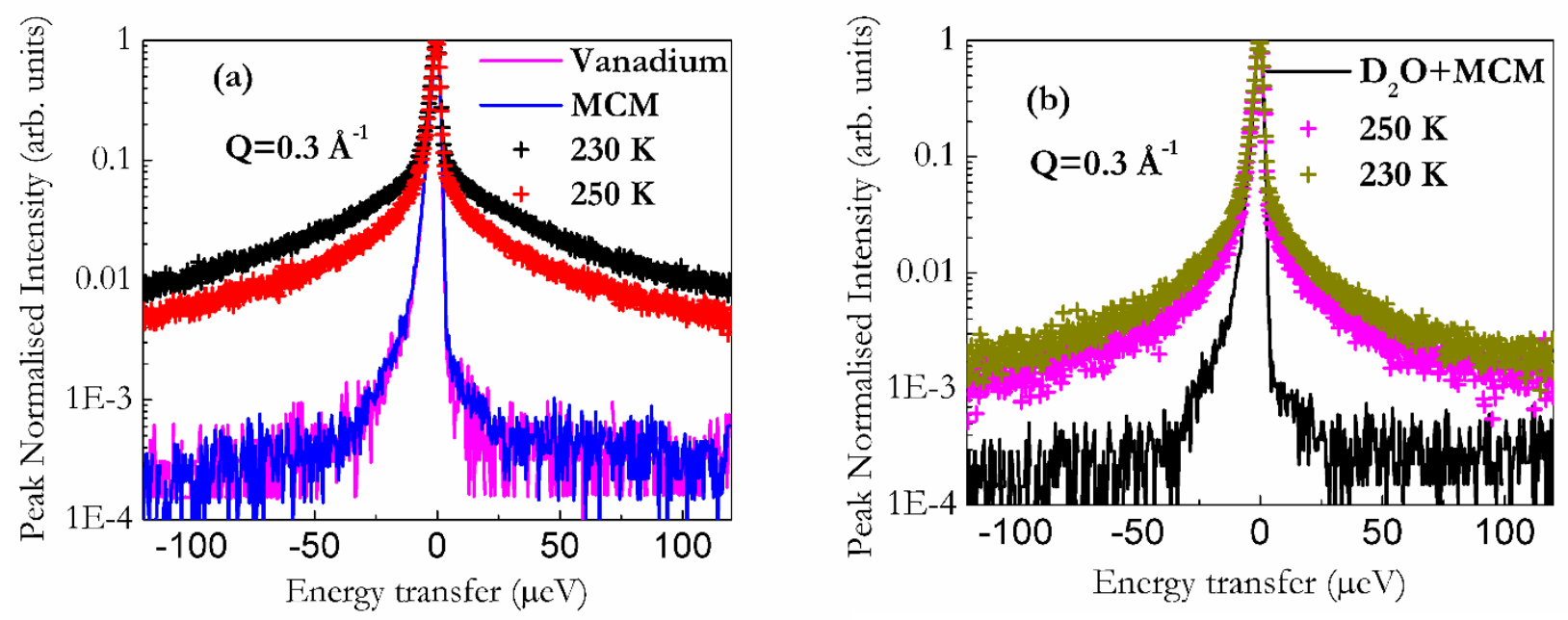

Figure 1. Peak normalised spectra obtained from the QENS experiments on propane in (a) dry, and (b) hydrated (with $\mathrm{D}_{2} \mathrm{O}$ ) MCM-41-S at $\mathrm{Q}=0.3 \AA^{-1}$ at two temperatures 230 and $250 \mathrm{~K}$. Also included in the plot are the spectra collected for a Vanadium and bare MCM-41-S measured at $300 \mathrm{~K}$ (shown in panel (a)) and empty hydrated MCM-41-S measured at $10 \mathrm{~K}$ (panel (b)). The effect of $\mathrm{D}_{2} \mathrm{O}$ on the mobility of propane in MCM-41-S can be seen as a suppression of quasielastic broadening (data shown with '+' symbols) in the panel (b) as compared to panel (a).

QENS data analysis was performed to quantify the suppression of propane motion in MCM-41-S upon addition of $\mathrm{D}_{2} \mathrm{O}$. As mentioned earlier, to obtain QENS data that represented only propane, the spectra for dry MCM-41-S were subtracted from the spectra for propane in MCM-41-S and the spectra for $\mathrm{D}_{2} \mathrm{O}$-loaded MCM-41-S were subtracted from the spectra for propane in $\mathrm{D}_{2} \mathrm{O}$ loaded MCM-41-S. It is common practice to describe the $S_{\text {inc }}(Q, \omega)$ as composed of an elastic and a quasielastic component along with a background. This $S_{i n c}(Q, \omega)$ is convoluted with the

29 The model $S_{\text {inc }}(Q, \omega)$ can thus be written as: 
31 In Eq. (7), the first term on the right side is the elastic contribution, which is approximated by a 32 delta function located at zero energy transfer; $A(Q)$ is the fraction of scattering that is elastic, which is called elastic incoherent structure factor $(E I S F)$. The second term on the right side is the quasielastic component, represented by a Lorentzian function centred at zero energy transfer with a half-width at half-maximum $(H W H M)$ of $\Gamma(Q)$. The third term is the background, while $R(Q, \omega)$ is the instrument resolution. Fitting the experimental spectra with Eq. (7) did not result in good

$S_{\text {inc }}(Q, \omega)=\left\{A(Q) \delta(\omega)+(1-A(Q))\left[L_{1}\left(\Gamma_{1}(Q), \omega\right)+L_{2}\left(\Gamma_{2}(Q), \omega\right)\right]+B(Q, \omega)\right\} \otimes R(Q, \omega)$ quality of fits for any propane spectra. Therefore, a combination of two Lorentzians was used to describe the quasi-elastic component for propane. The resulting fitting equation was

$$
S_{i n c}(Q, \omega)=\left\{A(Q) \delta(\omega)+(1-A(Q))\left[L_{1}\left(\Gamma_{1}(Q), \omega\right)+L_{2}\left(\Gamma_{2}(Q), \omega\right)\right]+B(Q, \omega)\right\} \otimes R(Q, \omega)
$$

Good quality fits were obtained using Eq. (8), shown in Figure 2. The need for two Lorentzians to describe the quasielastic part of the spectra of both dry and hydrated samples indicates the presence of two populations of propane molecules, which move at different time scales. The faster one of these populations is represented by the Lorentzian with a broader profile (large $\Gamma(Q)$ values), while the slower population is described by the Lorentzian with narrower profile (smaller $\Gamma(Q)$ values). The fitting parameters $A(Q), \Gamma_{l}(Q)$ and $\Gamma_{2}(Q)$ were analysed further, as discussed below.

Very low values of $A(Q)$ were obtained from the fits of all the experimental spectra. This indicated an absence of localised motion, or a population of immobile propane molecules in both dry and hydrated MCM-41-S. Fitting of Eq. (8) revealed $\Gamma_{l}(Q)<\Gamma_{2}(Q)$, where the former represents slow motion while the latter represents fast motion. The variation of $\Gamma_{l}(Q)$ and $\Gamma_{2}(Q)$ obtained from the fits of different propane spectra with $Q^{2}$ are shown in Figure 3. These variations are characteristic of a jump diffusion mechanism. In particular, the Singwi-Sjölander model of jump diffusion provides an adequate description of this behavior. In this model, motion occurs via jumps: wherein a molecule sits at a particular site for a time equal to the residence time, $\tau$, before jumping to another site, almost instantaneously. In this model, the $\Gamma(Q)$ varies according to the following relation $^{45}$ :

$57 \quad \Gamma(Q)=\frac{D Q^{2}}{1+D Q^{2} \tau}$

The variations of $\Gamma_{l}(Q)$ and $\Gamma_{2}(Q)$ were fitted with Eq. (9) and the obtained parameters - selfdiffusion coefficients $(D)$ and residence times $(\tau)$ are shown in Fig. 4. Effects of water on the 
60 dynamics of confined propane are reduction of the diffusion coefficients (top panels) and 61 corresponding lengthening of residence times (bottom panels) of both components in hydrated 62 samples. The enhancement of motions at higher temperature is seen in all cases except for the slow 63 component of the hydrated sample (indicating a counter-intuitive suppression of mobility at higher 64 temperature). However, we note that the error bars on both the diffusion coefficient and residence 65 time values are rather large making the interpretation of temperature effects on the experimental 66 data less certain. However, the variations of quantities with hydration is larger than the error bars 67 and suggest a clear suppression of propane mobility due to presence of water.
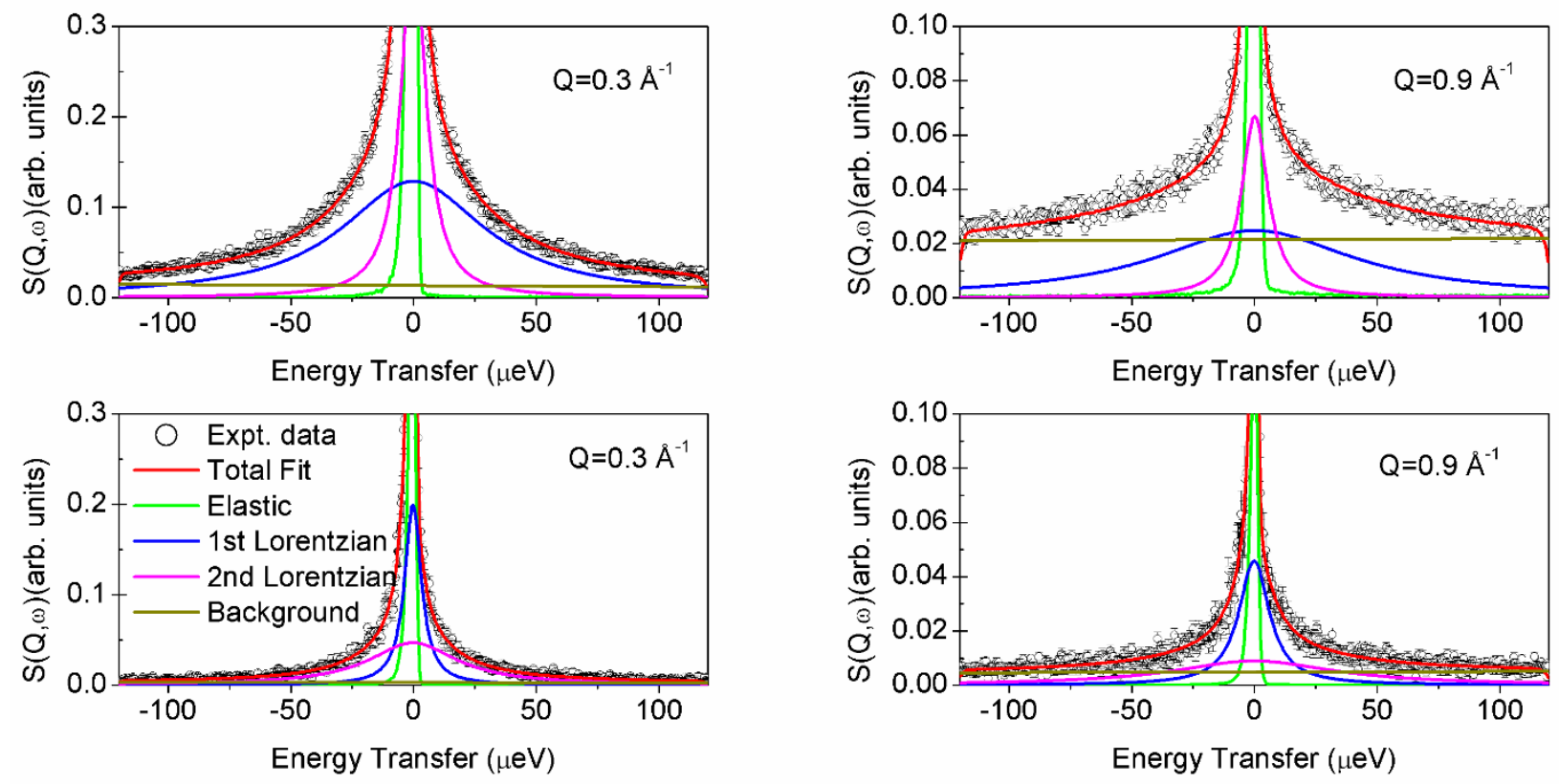

Figure 2. QENS spectra fitted with Eq. (8) for propane in dry (top) and hydrated (bottom) MCM-41-S at $250 \mathrm{~K}$. The left panels show the spectra at $Q=0.3 \AA^{-1}$, while the right panels show those at $Q=0.9 \AA^{-1}$. The experimental data are shown in symbols, the overall fits in red lines. 
74

75

76

77

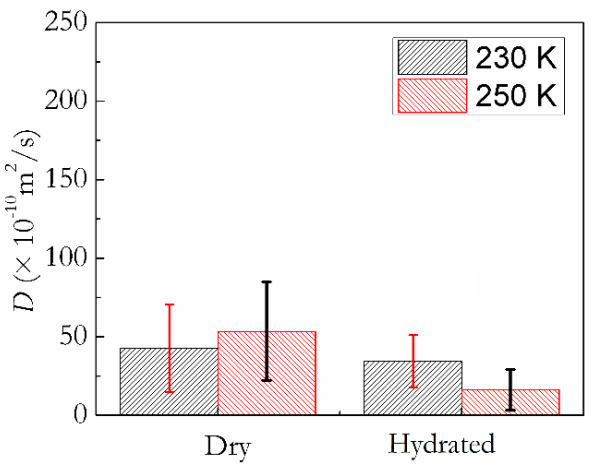

Slow Component

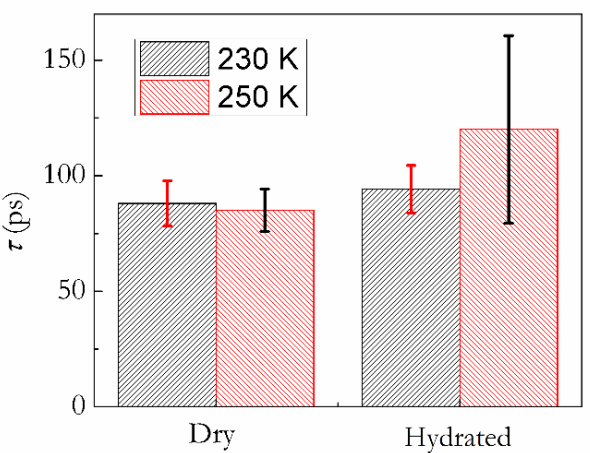

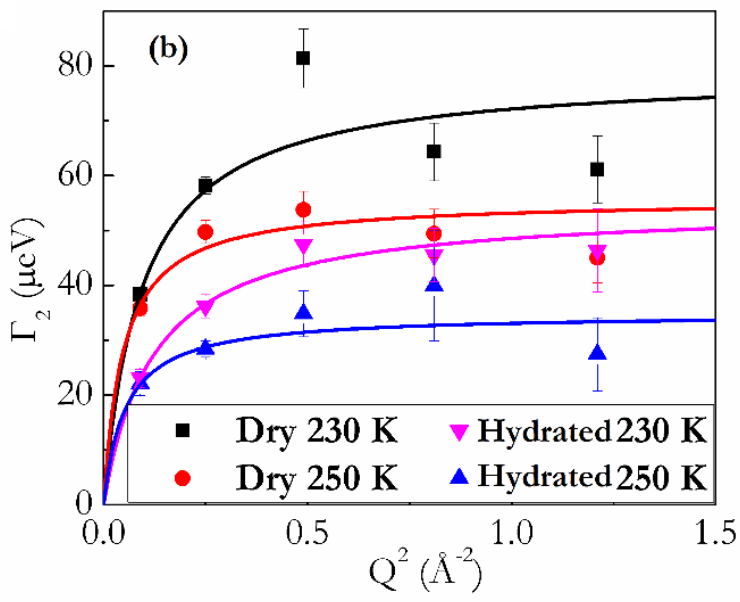

Figure 3. Variation of (a) $\Gamma_{l}(Q)$ and (b) $\Gamma_{2}(Q)$ obtained from fitting the QENS spectra of propane in dry and hydrated MCM-41-S at 230 and $250 \mathrm{~K}$, to Eq. (9). The solid lines show the fits of the $\Gamma(Q)$ variation to the jump diffusion model.

- Dry $230 \mathrm{~K}$

- Dry $250 \mathrm{~K}$

$\checkmark$ Hydrated $230 \mathrm{~K}$

$\triangle$ Hydrated $250 \mathrm{~K}$ $Q^{2}\left(\AA^{-2}\right)$

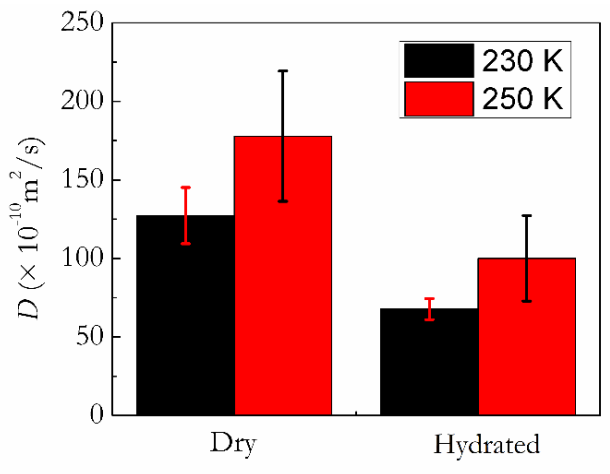

Fast Component

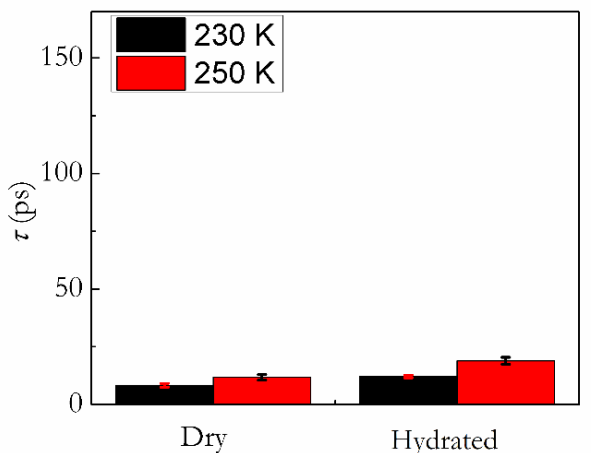

79 Figure 4. Values of $D$ (top) and $\tau$ (bottom) obtained from fitting of the variation of $\Gamma_{l}(Q)$ and $80 \quad \Gamma_{2}(Q)$ with Eq. (9) for propane in dry and hydrated MCM-41-S at 230 and $250 \mathrm{~K}$. The values in 81 the right panel are obtained from the fast component of motion, while those in the left panel are from the slow component. 


\subsection{Simulations}

Figure 5 shows the distribution of molecular propane density in the model MCM-41-S pore in the radial and axial directions at $230 \mathrm{~K}$. The corresponding atomic density distributions for oxygen atoms of water are shown in Figure S1 in the Supplementary Information. To calculate these density profiles, the centers of mass positions of all propane molecules are recorded at all times and averaged over 4-7 independent simulations. In the case of dry pore, propane molecules arrange themselves in a layer close to the pore surface, peaking at around $4.8 \AA$ from the pore axis. As a low amount of water is introduced in the pore $(\mathrm{H} 1)$, the peak in propane density is shifted towards the pore axis, as water molecules preferentially adsorb at the pore surface because of their polar nature. Even though the position of the molecular layer of propane is shifted by water, no other pronounced density peak can be seen. As more water is added to the pore $(\mathrm{H} 2)$, a new second propane layer begins to form close to the pore axis, at the expense of the layer closer to the pore surface. At higher water loadings ( $\mathrm{H} 3$ and $\mathrm{H} 4$ ), the layers close to the pore axis get denser, because water pushes away propane from the pore surface. Further, at high water contents the propane density profiles are characterised by sharper peaks. In the axial direction, shown in panel (b), the propane molecules are distributed evenly throughout the length of the dry pore as evidenced by an almost featureless flat distribution shown by the red curve. As water is added, a more uneven distribution can be observed (green curve, H1) with more peaks in the central region. As more water is present, the distribution exhibits sharper and more numerous peaks. At highest water content (H4), the large amount of water forces propane molecules to cluster together, yielding layers along the axial direction. A tendency of 'ordering' in the orientational distribution of propane molecules is also observed at high water content (see Supplementary Information, Figure $\mathrm{S} 2$ ). This can be due to the propane molecules being trapped by water molecules, suggesting that ordering is promoted in this system by low mobility of propane molecules. A similar tendency of nanopore ordering due to low mobility has also been observed for acetone, acetaldehyde and acetonitrile in $\mathrm{ZSM}-5^{25}$. 

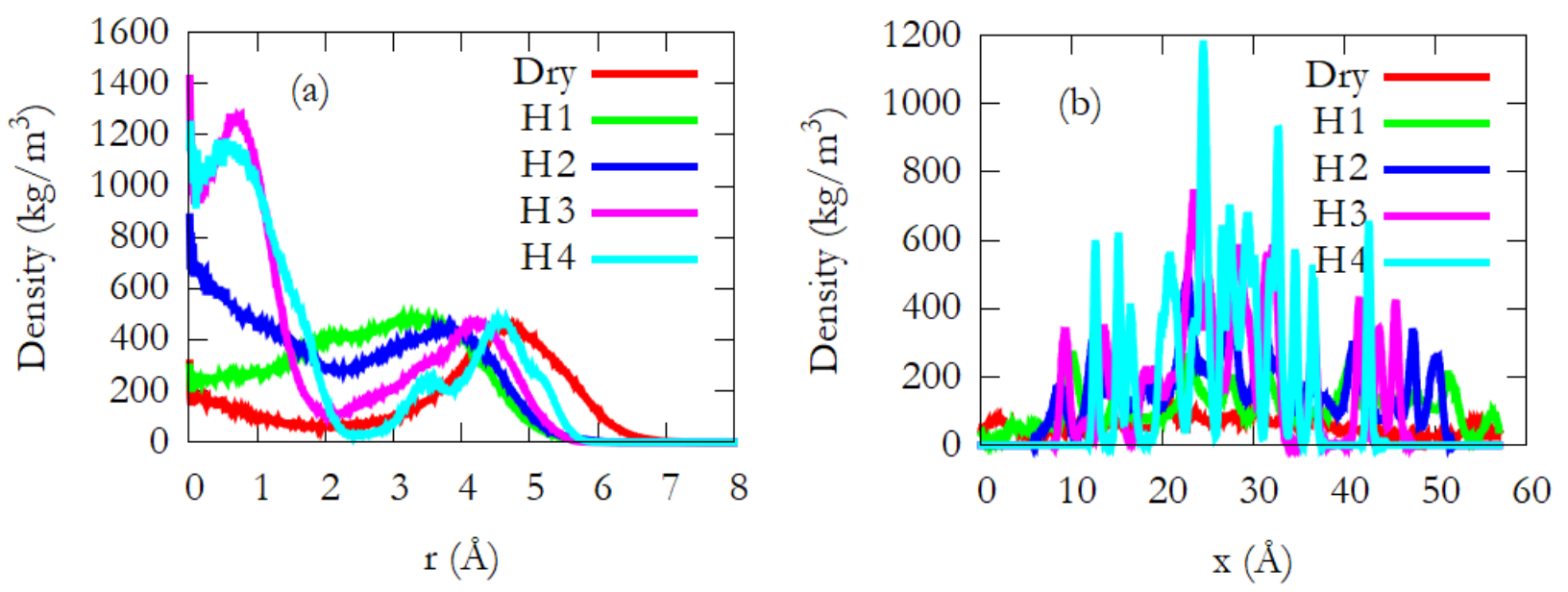

Figure 5. Density distribution of propane molecules in a model MCM-41-S pore along the (a) radial and (b) axial directions. The pore is centered at $r=0 \AA$ while in the axial direction the simulation cell boundaries are located at $\mathrm{x}=0$ and $56.9 \AA$.

Figure 6 (a) shows the mean squared displacement (MSD) of the center of mass of propane molecules in the model MCM-41-S pore at $230 \mathrm{~K}$. The results are shown as a function of water content. Similar results obtained at $250 \mathrm{~K}$ for propane can be found in the Supplementary Information (Figure S3). The MSD of propane at long times gets suppressed with water content in the pore, indicating suppression of mobility. At the highest water content (H4), the MSD plateaus quickly to a constant value, and does not show any appreciable increase after about $0.5 \mathrm{ps}$. The overall MSD at $230 \mathrm{~K}$ in the case of dry sample has also been resolved in three Cartesian directions. As the pore is oriented along the Cartesian X-direction, the overall MSD is almost completely described by the MSD along X-direction (see Supplementary Information, Figure S4). At very short times, the values of MSD along all directions are similar. This is the ballistic regime, where the molecules move freely before colliding against other molecules. At intermediate times, because of layered structure of the fluids, a typical molecule is more likely to collide with another molecule along the radial direction in the pore (Cartesian $\mathrm{Y}$ or $\mathrm{Z}$ ) and so the value of MSD along these directions is suppressed because of more frequent collisions. At longer times, the finite size of the pore along the radial direction puts an absolute limit on the mobility of molecules in this direction, and the molecules are unable to move a squared distance of more than $\sim 20 \AA^{2}$. This limit is reached at $\sim 50$ ps. After this time, motion along the pore axis is solely responsible for the increase of MSD 
131 with time and the corresponding motion is represented by 1-D diffusion. The MSD along the pore 132 axis for all systems at $230 \mathrm{~K}$ is shown in Figure 6 (b).

133
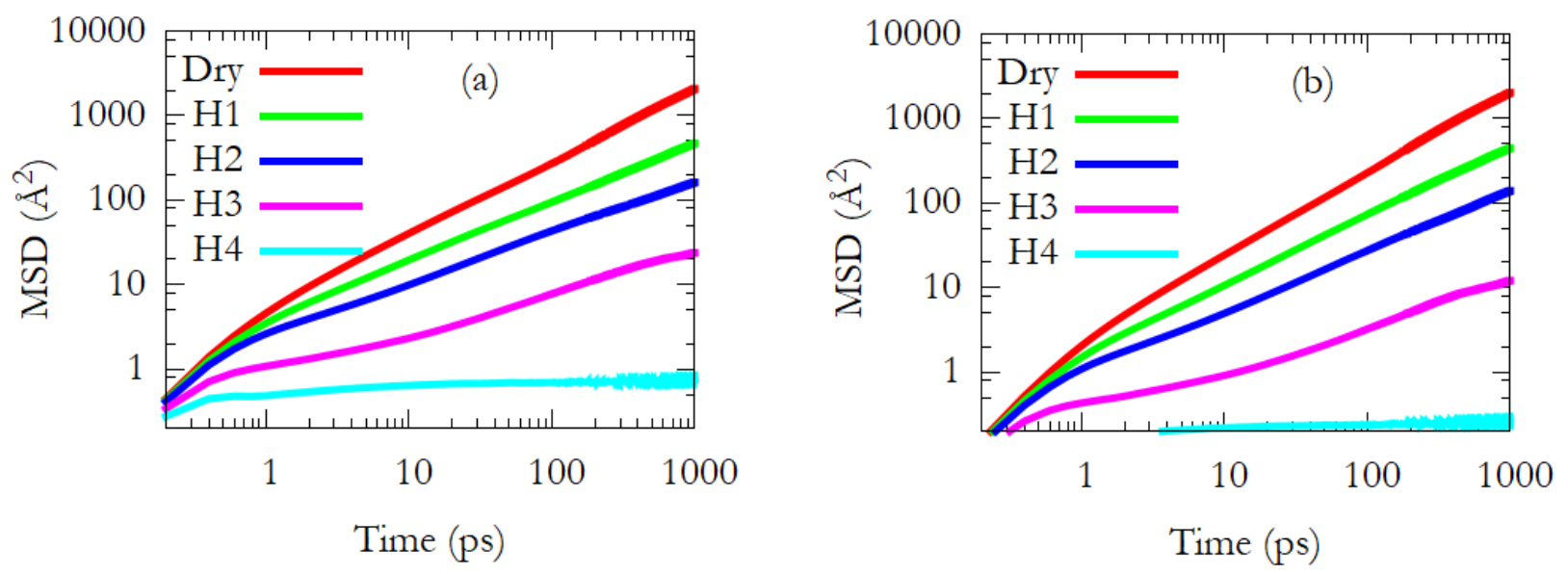

Figure 6 (a) Mean squared displacements of the center of mass of propane molecules in model MCM-41-S pore with different moisture contents at $230 \mathrm{~K}$. (b) MSD along the axial direction

1-dimensional diffusion coefficients have been obtained from the long-time slope of the MSD along X-direction vs time plots using Eq. 1 and are listed in Table 2. The diffusive nature of motion

141 at long times was ascertained by examining the slope of $\ln (\mathrm{MSD})$ vs $\ln (t)$ plot. The slopes of these

142 plots for all the systems, except H4, were found to be close to 1 at long enough times, indicating 143 diffusive motion. As the motion of propane molecules in the simulation H4 is severely constrained 144 and not diffusive, no reliable diffusion coefficient is obtained from this simulation. Dynamics of 145 water at the temperatures reported here was severely constrained. However, water molecules in all 146 the systems except H4 exhibited mobility. Water mobilities were reduced by a factor of $\sim 10$ 147 compared to those at $300 \mathrm{~K}$ reported earlier ${ }^{35}$ (see Supplementary Information, Table S1). 
Table 2. 1-dimensional diffusion coefficients of propane in the systems listed in Table 1. For the system H4 it was not possible to extract reliable values for the propane self-diffusion coefficient because in this system the motion is sub-diffusive even at long times.

\begin{tabular}{|l|l|l|}
\hline \multirow{2}{*}{ Sample } & \multicolumn{2}{|l|}{$\mathrm{D}\left(\times 10^{-10} \mathrm{~m}^{2} / \mathrm{s}\right)$} \\
\cline { 2 - 3 } & $230 \mathrm{~K}$ & $250 \mathrm{~K}$ \\
\hline Dry & $99.5 \pm 3.1$ & $122.1 \pm 3.4$ \\
\hline H1 & $14.8 \pm 0.6$ & $20.4 \pm 0.7$ \\
\hline H2 & $6.1 \pm 0.3$ & $7.6 \pm 0.3$ \\
\hline H3 & $0.5 \pm 0.1$ & $0.7 \pm 0.2$ \\
\hline H4 & - & - \\
\hline
\end{tabular}

As discussed above, to properly connect with the QENS experiments, it is desirable to extract the TISF from the simulated trajectories. Figure 7 shows the TISF at $\mathrm{Q}=0.99 \AA^{-1}$ calculated from different simulations at $230 \mathrm{~K}$. Higher water loading results in a slower TISF decay, i.e., slower motions of propane. The time range accessible with the BASIS instrument used in the QENS experiment is highlighted in color in Figure 7. Within this time range, the TISF for most compositions appears to exhibit at least two behaviors. These two behaviors can best be interpreted by consideration of the existence of two dynamical populations of propane molecules, which is consistent with the experimental data. Expanding on this comparison further, the TISF calculated from simulations for dry and H1 compositions can be fitted with exponential decay functions in the time range from 3 to $200 \mathrm{ps,}$, accessible to instrument, in order to estimate the time scales involved in the motion represented by these functions. As noted earlier (Section 3), these time scales can be converted to corresponding $\Gamma^{s}{ }_{i}(Q)(i=1,2$; superscript 's' is used to indicate quantities obtained from the simulation data) using Eq. (5) and can be compared with $\Gamma_{l}(Q)$ and $\Gamma_{2}(Q)$ obtained from the QENS experiments. As the quasielastic spectra were fitted with two Lorentzians, the TISF were fitted with two exponential decay functions as well. This translates to fitting $\ln$ (TISF) vs. time plot with a combination of two linear functions ${ }^{\dagger}$. The slope of these two lines yields the time scales $(\tau)$.

\footnotetext{
${ }^{+}$TISF $\sim \mathrm{e}^{(-t / \tau)}$ is equivalent to $\ln [\mathrm{TISF}] \sim(-\mathrm{t} / \tau)$ and hence $\ln [\mathrm{TISF}]$ vs time is a straight line with slope $=(-1 / \tau)$
} 


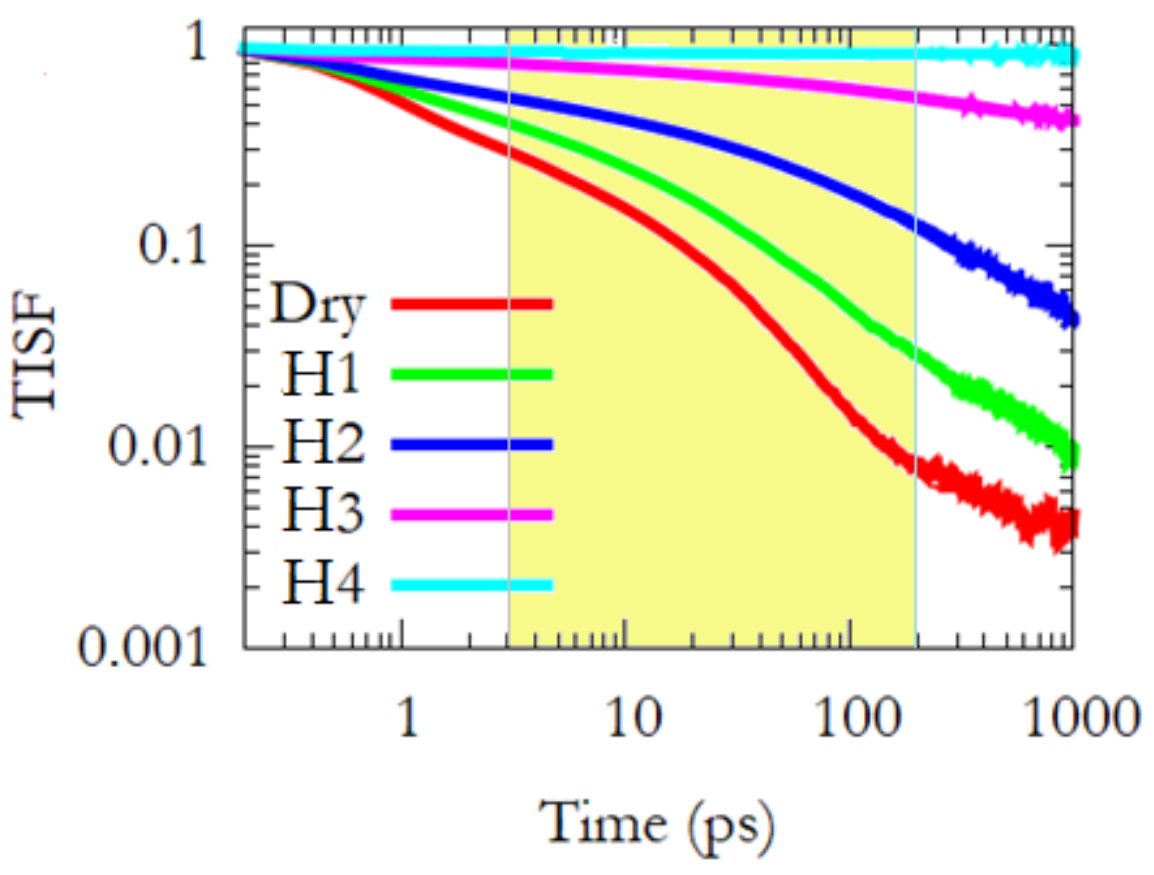

Figure 7. TISF as a function of time calculated from the trajectories of center of mass of propane molecules using Eq. (2) from simulations conducted at $230 \mathrm{~K}$. All TISFs shown are calculated at $\mathrm{Q}=0.99 \AA^{-1}$. The time range accessible to the instrument used in the experiment is highlighted in colour.

Representative $\ln$ (TISF) vs. time curves for Dry and H1 systems at 230K are shown in Figure 8. The initial, very fast, decay that lasts for $\sim 1$ ps represents very fast motion and is out of the time range of the instrument. Beyond that, the $\ln$ (TISF) can be divided into two regions with varying slopes. To maintain consistency, all $\ln (\mathrm{TISF})$ vs. time plots were fitted within the two ranges of 360 ps and 100-200 ps with linear functions. The $\Gamma^{5} i(Q)(i=1,2)$ obtained from the fits for the two compositions are shown in Figure 9 as functions of $Q^{2}$. As in the experiments, the variation of $\Gamma^{\mathrm{S}}{ }_{i}(Q)$ with $Q^{2}$ is further fitted with a jump diffusion model (Eq. (9)) to obtain diffusion coefficients and residence times. The values of these parameters obtained from the fits are listed in Table 3. The magnitude of the parameters is consistent with those obtained from the experiments. The small quantitative differences result from the difference in compositions used in the experiment and the simulations, along with other typical computational limitations, including the implementation of a generic force field which has not been optimised to reproduce the experiments presented here. 

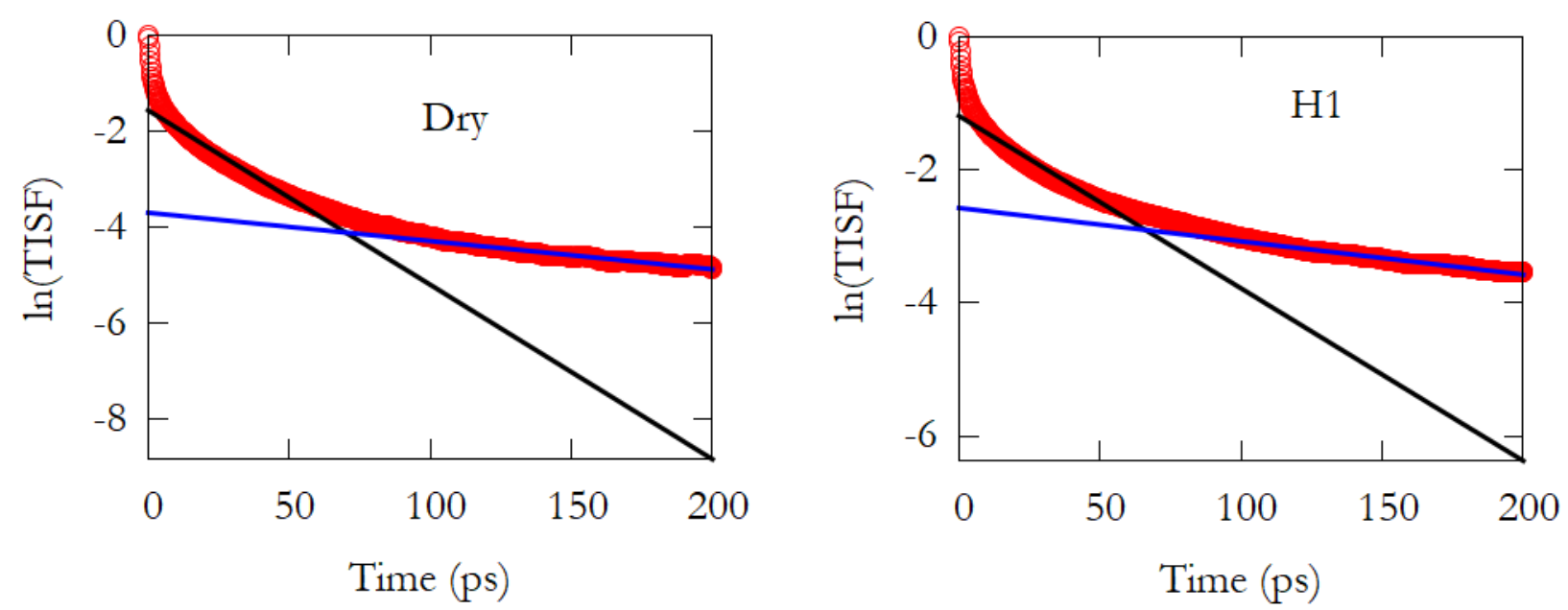

190 Figure 8. Fits of the simulation derived $\ln (\mathrm{TISF})$ vs. $t$ curves in two different time ranges (3-60 191 ps and 100-200 ps) with linear functions. The slope of the fitted linear functions are proportional 192 to $\Gamma_{i}^{s}(Q)(i=1,2)$, which can be directly compared with the HWHM of the Lorentzians representing the quasielastic width $\left(\Gamma_{1}(Q)\right.$ and $\Gamma_{2}(Q)$ shown in Figure 3) as obtained from the QENS experiment.
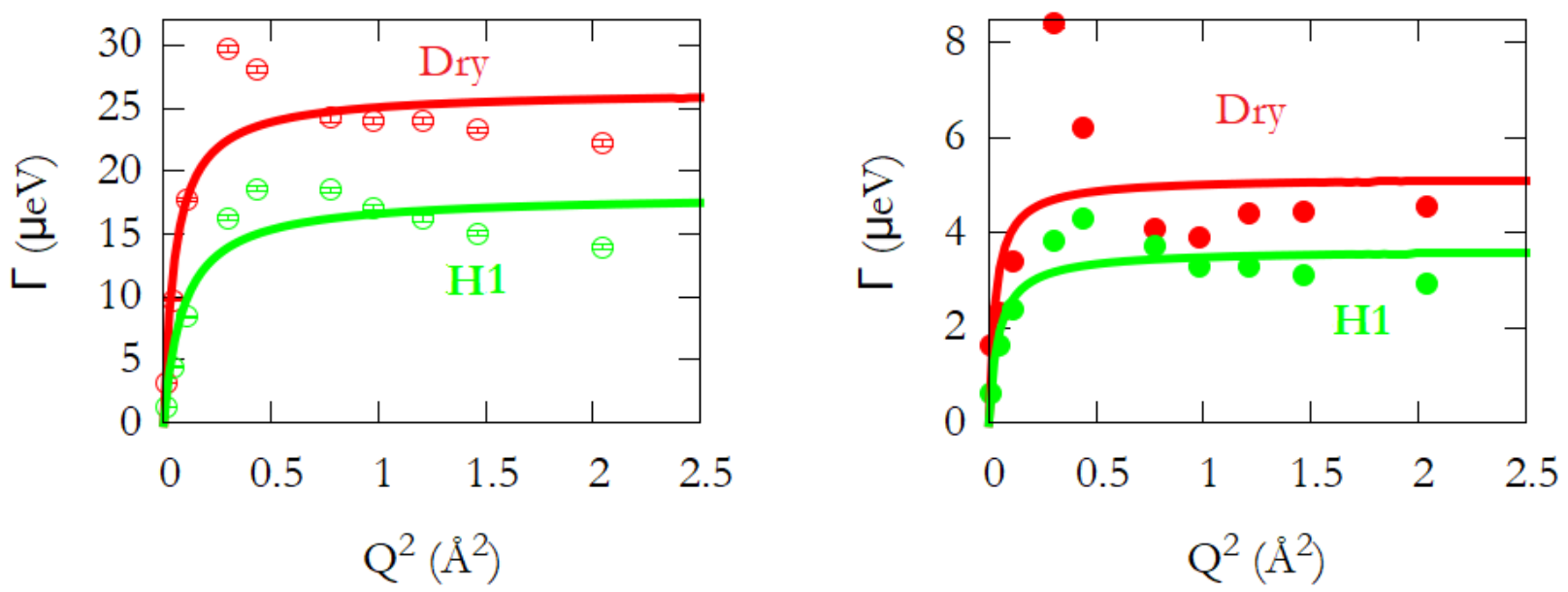

197 Figure 9. Energies $\left(\Gamma_{i}^{\mathrm{S}}(Q)(i=1,2)\right)$ involved in the motion represented by the simulation-derived 198 TISF in the time range 3-60 ps (left) and 100-200 ps (right). The solid lines are fits to the data 199 obtained using the jump diffusion model. The fast component of the motion is represented by $200 \Gamma_{1}{ }_{1}(Q)$ in the left panel, while the slow component is represented by $\Gamma_{2}^{\mathrm{s}}(Q)$ in the right panel. In the right panel, the error bars are smaller than the symbols used to show the data. 
Table 3. Parameters of jump diffusion obtained from the fitting shown in Figure 9.

\begin{tabular}{|l|l|l|}
\hline System-Component & $\mathbf{D}\left(\times \mathbf{1 0}^{-10} \mathbf{m}^{\mathbf{2}} / \mathbf{s}\right)$ & $\boldsymbol{\tau}(\mathbf{p s})$ \\
\hline Dry-Fast & $77.0 \pm 30.4$ & $25.0 \pm 1.7$ \\
\hline Dry-Slow & $26.5 \pm 24.5$ & $127.8 \pm 17.3$ \\
\hline H1-Fast & $30.5 \pm 11.4$ & $36.3 \pm 2.8$ \\
\hline H1-Narrow & $12.4 \pm 5.2$ & $181 \pm 12.6$ \\
\hline
\end{tabular}

204

205 Figure 10 shows a comparison of the RCF calculated from simulations of different composition.

206 The time range accessible to BASIS is highlighted in color. The effect of water content on the 207 rotational motion of propane is qualitatively the same as that on the translational motion, i.e., water 208 suppresses rotational motion. Further, the RCF for all compositions, except the two highest water 209 contents, decay completely before the lower limit of the time scales accessible with the instrument. 210 This shows that if a QENS experiment were carried out on these compositions using BASIS, the 211 rotational motion of propane will not contribute to the quasielastic spectra in all, but the two 212 highest water content compositions.

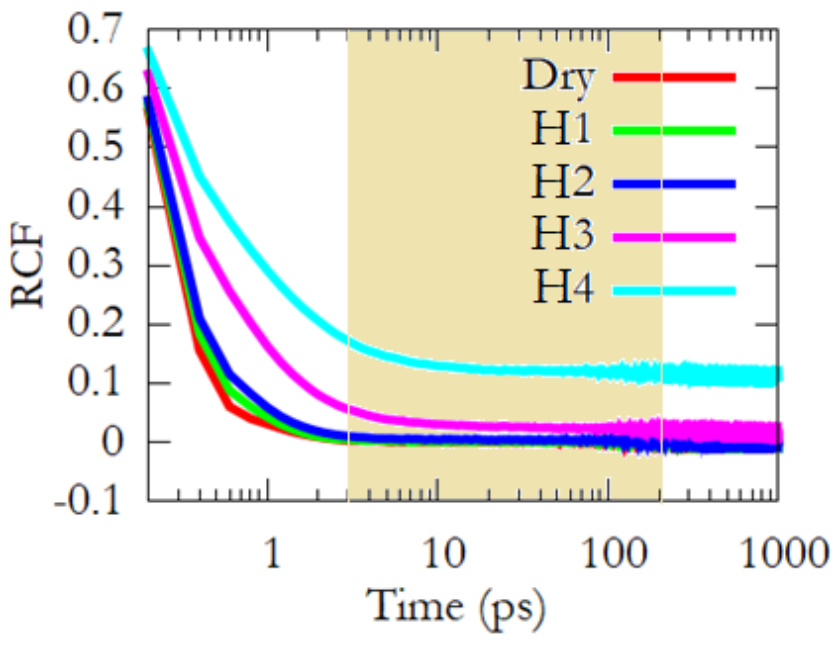

215 Figure 10. Rotational correlation function (RCF) of propane in different simulations. The time 216 range accessible to the instrument BASIS is highlighted in colour. 


\section{Discussion}

218 We report diffusion coefficients of propane confined in dry and hydrated pores of MCM-41-S using experimental and computational approaches. Although quantitative differences exist between the results from the two techniques, the order of magnitudes and trends in the selfdiffusion coefficient observed are very similar. For bulk propane at saturation vapor pressure,

222 Schmid et al. ${ }^{46}$ reported self-diffusion coefficients of $54.4 \times 10^{-10}$ and $73.9 \times 10^{-10} \mathrm{~m}^{2} / \mathrm{s}$ at $228 \mathrm{~K}$ and

$223249 \mathrm{~K}$, respectively. The values obtained here for propane confined in a dry MCM-41-S pore are 224 comparable to these values. We note that in our experiments, confined propane remains in the 225 vapor phase even at the lower temperatures probed. The effect of confinement due to the MCM41-S pores seems to correspond to an increase in pressure, which would imply an increase in density for the confined fluid, consistent with many simulation results ${ }^{47}$.

The values of residence times obtained experimentally and from the simulation-derived TISFs (Figure 4 and Table 3) are rather high, especially for the slower component of motion and in the presence of water. For example, compared to ethane in $\mathrm{CPG}^{10}$ and propane in silica aerogel ${ }^{13}$, the residence times obtained for propane in MCM-41-S are a factor of 3-4 higher for the fast component and, in some cases, even 2 orders of magnitude higher for the slow component. We note that the diffusive properties of propane in silica aeroge ${ }^{13}$ were measured using the same instrument used here, implementing the same settings of elastic resolution and energy window. The slow component measured in the present study thus exhibits remarkably high residence times, implying very strong intermolecular interactions, especially when water is present. The slow component might represent a motion where a propane molecule shuttles within a small region for a long time (i.e., the residence time), and then occasionally overcomes a high potential barrier to jump to a distant site. The shuttling motion in between jumps is too fast to be captured by the BASIS instrument. It is this fast shuttling motion that is represented by the very short time fast decay of the TISF smaller than $\sim 3$ ps shown in Figure 8 .

242 Although the residence times of the two populations of propane molecules are very different from each other, the differences in the corresponding diffusion coefficients are relatively small. However, the existence of two different motions is indicated by an analysis of the scattering law 245 or the intermediate scattering functions, while the simulated mean square displacement profiles yield only an averaged result. Because the $Q$ variations of the energies involved in propane motions 
$247\left(\Gamma_{i}(Q)\right.$ from the experiments and $\Gamma_{i}^{s}(Q)$ from the simulations) indicate diffusion occurring via 248 jumps, the results were fitted with the Singwi-Sjölander jump diffusion model. In this model, the variation of $\Gamma(Q)$ with $Q^{2}$ exhibits an initial fast growth close to a straight line, followed by a plateau. The slope of the initial growth period determines the diffusion coefficient, while the value 251 at the plateau determines the residence times. As the number of data points in the $D$-determining low $Q$ region is small in both simulations and experiments, the estimated $D$ is more prone to errors than $\tau$. This problem is further aggravated in the slow component, as the $\Gamma(Q)$ values obtained representative of this component are themselves prone to larger errors. This is because of the difficulty involved in measuring a smaller quantity, especially in the presence of a larger quantity. This experimental uncertainty might explain the counter-intuitive enhancement of motion at 230 $\mathrm{K}$ compared to $250 \mathrm{~K}$ for the slow component of the hydrated sample, while the temperature variation by the MSD-derived $D$ follows expectations.

The effect of water on the dynamics of confined propane is similar to that reported at $300 \mathrm{~K}$ in an earlier publication ${ }^{35}$. However, the effect reported here is very different to that reported for $\mathrm{CO}_{2}$ on the dynamics of confined ethane ${ }^{10}$, propane ${ }^{13}$, butane ${ }^{30}$ and octane ${ }^{29} \cdot \mathrm{CO}_{2}$ was found to enhance the mobility of the confined hydrocarbons in all these studies. This enhancement ('molecular lubrication') was explained by preferential adsorption of $\mathrm{CO}_{2}$ to the pore walls, which results in the $\mathrm{CO}_{2}$ molecules pushing the hydrocarbon molecules away from the pore surface, and thereby reducing their energy barrier for diffusion ${ }^{30}$. Also in the present study, water was found to displace

266 propane molecules away from the pore wall. However unlike in the case of $\mathrm{CO}_{2}$, the displacement 267 of propane from the pore surface by water resulted in a lowering of the propane diffusion coefficient, possibly because of the formation of barriers to propane diffusion due to the formation of hydrogen-bonded networks of water molecules that span the entire pore width, as discussed below.

271 The size and geometry of the pores seem to be important factors, as well as the strong water-water 272 preferential interactions (i.e., hydrogen bonds) ${ }^{48}$. The pore size of MCM-41-S sample used here 273 is smaller than the pores used in the experimental studies reported in refs. 10 and 13 by factors of $274 \sim 5$ and 12, respectively. Although the pore size used in refs. 29 and 30 of $\sim 2 \mathrm{~nm}$ was comparable 275 to the present case $(\sim 1.5 \mathrm{~nm})$, the pore geometry was significantly different. In the MCM-41-S 276 pore, the sorbents are constrained to move within a cylinder of diameter $1.5 \mathrm{~nm}$, resulting in a free 
277 motion only along one dimension. In refs. 29 and 30, a slit pore was used, hence the fluid motion

278 was constrained in one direction and free within a 2-dimensional plane. This means that both the 279 cylindrical pore geometry as well as its smaller diameter result in a more crowded environment 280 for the adsorbate. These geometrical effects favour the formation of water bridges within the 281 narrow pores, especially at high water loadings. Thus, although water displaces propane from the 282 pore surface, the overall effect of increasing water content is to increase crowding and strongly 283 suppressing the mobility of confined propane.

284 Our results show that the rotational motion of confined propane molecules also exhibits 285 suppression by hydration, which is possibly due to the restricted pore volume available to propane 286 once water enters the MCM-41-S pores. The simulation data show that the rotational motions of 287 propane in all but the two highest water contents are too fast to contribute to the experimental 288 quasielastic spectra. This is consistent with the experiments, where the small values of EISF obtained from fitting the QENS spectra of all samples indicate absence of any localized motion, including the rotational motion. Further, in the two highest water content simulations, the RCF of propane do not decay to zero even after $1 \mathrm{~ns}$. This residual correlation in the orientation of the propane molecules at times separated by long intervals indicates that propane molecules are unable to trace the entire orientational space available to them. This is due to water molecules, which block the rotation of propane at high water contents. MD study of this system at higher temperature showed that water blocks propane mobility by forming molecular bridges ${ }^{35}$. This reduced mobility 296 in translational motion gives rise to inhomogeneities in the density distribution of propane along 297 both axial as well as radial directions at high water contents (Figure 5). A similar reduction in 298 orientational motion gives rise to orientational ordering of propane molecules at high water 299 contents (see Fig. S2 in the Supplementary Information). It is likely that the elongated geometry 300 of one propane molecule enhances the reduction of its rotational dynamics when water content 301 increases.

302 QENS experiments have been conducted at conditions such that the confined propane always 303 remains in the vapour state. Although the experimental temperature is lower than what is expected 304 in the subsurface, the thermodynamic state of propane is similar (i.e., propane remains in the vapor 305 phase). Therefore, the results presented are of relevance to practical situations encountered in the 
306

307

308

309

310

311

312

313

314

315

316

317

318

319

320

321

322

323

324

325

326

327

328

329

330

331

332

333

subsurface, where hydrocarbons can be found in water-bearing-porous networks composed of silica-rich substrates.

\section{Conclusions}

We have used a combination of quasielastic neutron scattering (QENS) experiments and molecular dynamics (MD) simulations to study the effect of water on the dynamics of propane confined in MCM-41-S cylindrical pores of diameter $1.5 \mathrm{~nm}$. Both experiments and simulations show that presence of water suppresses the mobility of propane. MD simulations show that this effect is dependent on the amount of water in the pore. At the highest water content, both structure and dynamics, translational as well as rotational, of confined propane are severely constrained. Water is found to displace propane molecules from the pore surface. A similar mechanism involving $\mathrm{CO}_{2}$ has been found to enhance the diffusivity of several hydrocarbons, including propane. In the present case though, the effects of displacement of propane from the pore surfaces are countered by an increased molecular crowding, which is due to a combination of small pore diameter, cylindrical pore geometry, and strong water-water hydrogen bonds. The resultant effect is an overall suppression of propane mobility. The mechanism of propane diffusion does not however seem to be affected by the presence of water, at least at moderate water loadings. Our simulations suggest that above a water-loading threshold propane becomes trapped within the hydrated pores, at least for the time scales accessible to our MD simulations. Although the measurements reported here were made at low temperatures of 230 and $250 \mathrm{~K}$, propane was at low pressure and in the vapor phase. Thus, the present study could have implications for the subsurface environment where hydrocarbons in vapor phase are found trapped in water saturated porous networks even at geologic temperatures.

\section{Acknowledgements}

The quasielastic neutron scattering experiments reported here were carried out at the backscattering instrument BASIS at spallation neutron source (SNS), a US Department of Energy 
334 (DOE) office of science user facility operated by the Oak Ridge National Laboratory (ORNL). 335 S.G., T.T.B.L., A.S., T.L. and D.C., thank the financial support from U.S. Dept. of Energy (DOE), 336 Office of Science, Office of Basic Energy Sciences, Division of Chemical Sciences, Geosciences 337 and Biosciences, Geosciences Program under grant DE-SC0006878 to Ohio State University. 338 Material synthesis, experiment planning and measurements of experimental data, and contribution 339 to manuscript preparation by G.R., Z. Q., and SD were supported by the U.S. Department of 340 Energy, Office of Science, Office of Basic Energy Sciences, Chemical Sciences, Geosciences and 341 Biosciences Division. A.S. and T.T.B.L. are also grateful to partial financial support from the 342 Science4CleanEnergy European research consortium funded by European Union's Horizon 2020 343 research and innovation programme, under grant agreement No. 764810 (S4CE). S. G. also thanks 344 the computing resources provided by the Deep Carbon Observatory cluster hosted by Rensselaer 345 Polytechnic Institute.

\section{Conflict of Interest}

The authors declare that there is no conflict of interest.

\section{References}

351 [1] Cole, D. R., Mamontov, E. and Rother, G. in Liang, L., Rinaldi, R. and Schoeber, H., (Eds.)

352 Neutron Applications in Earth, Energy, and Environmental Sciences, 2009, 547-570.

353 [2] Wang, H., Wang, L., He, S., \& Xiao, F. S. "Enhancement of Catalytic Properties by 354 Adjusting Molecular Diffusion in Nanoporous Catalysts." In C. Song (Ed.) Advances in 355 Catalysis, Vol. 62, 1-46, Academic Press, 2018.

356 [3] Demontis, P., Suffritti, G. B. Structure and dynamics of zeolites investigated by molecular 357 dynamics. Chem. Rev., 1997, 97(8), 2845-2878.

358 [4] Hahn, K., Karger, J. and Kukla, V. Single-File Diffusion Observation, Phys. Rev. Lett., 1996, $35976,2762-2765$. 
360 [5] Bhide, S. Y. and Yashonath, S. Dependence of the Self-Diffusion Coefficient on the Sorbate 361 Concentration: A Two-Dimensional Lattice Gas Model With and Without Confinement, $J$.

362 Chem. Phys., 1999, 111, 1658-1667.

363 [6] Bhide, S. Y. and Yashonath, S. Types of Dependence of Self-Diffusivity on Sorbate 364 Concentration in Parameter Space: A Two-Dimensional Lattice Gas Study, J. Phys. Chem. B, $3652000,104,2607-2612$.

366 [7] Gautam, S., Ok, S., and Cole, D. R. Structure and Dynamics of Confined C-O-H Fluids 367 Relevant to the Subsurface: Application of Magnetic Resonance, Neutron Scattering and 368 Molecular Dynamics Simulations, Front. In Earth Sc. 2017 5:43. doi: 10.3389/feart.2017.00043

369 [8] Cole, D. R., Grszkiewicz, M. S., Simonson, J. M., Chialvo, A. A., Melnichenko, Y. B., 370 Wignall, G. D., Lynn, G. W., Lin, J. S., Habenschuss, A., Gu, B., et al., "Influence of Nanoscale 371 Porosity on Fluid Behavior” In: Water-Rock Interaction, 737-740, (Wanty, R. B. and Seal II, R. 372 R. Eds.) Taylor \& Francis, London 2004.

373 [9] Chathoth, S. M., Mamontov, E., Melnichenko, Y. B. and Zamponi, M. Diffusion and 374 Adsorption of Methane Confined in Nano-Porous Carbon Aerogel: A Combined Quasi-Elastic 375 and Small-Angle Neutron Scattering Study, Micro. Meso. Mater., 2010, 132, 148-153.

376 [10] Patankar, S., Gautam, S., Rother, G., Podlesnyak, A., Ehlers, G., Liu, T., Cole, D. R., and 377 Tomasko, D. L. Role of Confinement on Adsorption and Dynamics of Ethane and an Ethane$378 \mathrm{CO}_{2}$ Mixture in Mesoporous CPG Silica, J. Phys. Chem. C 2016, 120, 4843-4853.

379 [11] Gautam, S., Liu, T., Patankar, S., Tomasko, D., \& Cole, D. Location dependent orientational 380 structure and dynamics of ethane in ZSM5. Chem. Phys. Lett., 2016, 648, 130-136.

381 [12] Gautam, S., and Cole, D., Molecular Dynamics Simulation Study of Meso-Confined 382 Propane in $\mathrm{TiO}_{2}$, Chem. Phys. 2015, 458, $68-76$.

383 [13] Gautam, S., Liu, T., Rother, G., Jalarvo, N., Mamontov, E., Welch, S., Sheets, J., Droege, 384 M., and Cole, D. R. Dynamics of Propane in Nanoporous Silica Aerogel: A Quasielastic Neutron 385 Scattering Study, J. Phys. Chem. C, 2015, 119, 18188-18195. 
[14] Stepanov, A. G., Shegai, T. O., Luzgin, M. V., and Jobic, H. Comparison of the dynamics of n-hexane in ZSM-5 and 5A zeolite structures. The European Physical Journal E, 2003, 12, 5761.

[15] Gautam, S., Mitra, S., Mukhopadhyay, R. and Chaplot, S. L. Diffusion of Acetylene inside Na-Y Zeolite: Molecular Dynamics Simulation Studies, Phys. Rev. E, 2006, 74, 041202.

[16] Sharma, V. K., Gautam, S., Mitra, S., Rao, M. N., Tripathi, A. K., Chaplot, S. L. and Mukhopadhyay, R., Dynamics of Adsorbed Hydrocarbon in Nanoporous Zeolite Framework, $J$. Phys. Chem. B, 2009, 113, 8066-8072.

[17] Gautam, S., Sharma, V. K., Mitra, S., Chaplot, S. L., \& Mukhopadhyay, R. Rotational dynamics of propylene in ZSM-5 zeolitic frameworks. Chem. Phys. Lett., 2011, 501, 345-350.

[18] Gautam, S., Mitra, S., Sayeed, A., Yashonath, S., Chaplot, S. L., \& Mukhopadhyay, R. Diffusion of 1, 3-butadiene adsorbed in Na-Y zeolite: Neutron scattering study. Chem. Phys. Lett., 2007, 442, 311-315.

[19] Gautam, S., Mitra, S., Chaplot, S. L. and Mukhopadhyay, R. Dynamics of 1,3-Butadiene Adsorbed in Na-Y Zeolite: MD Simulation Study, Phys. Rev. E, 2008, 77, 061201.

[20] Thalladi, V. R., \& Boese, R. Why is the melting point of propane the lowest among nalkanes?. New J. Chem., 2000, 24, 579-581.

[21] Mukhopadhyay, R., Sayeed, A., Mitra, S., Kumar, A. V. A., Rao, M. N., Yashonath, S. and Chaplot, S. L. Rotational Dynamics of Propane in Na-Y Zeolite: A Molecular Dynamics and Quasielastic Neutron Scattering Study, Phys. Rev. E, 2002, 66, 061201

[22] Le, T., Striolo, A., and Cole, D. R. Propane Simulated in Silica Pores: Adsorption Isotherms, Molecular Structure, and Mobility, Chem. Eng. Sci. 2015, 121, 292-299.

[23] Gautam, S., Le, T., Striolo, A., and Cole, D. Molecular dynamics simulations of propane in slit shaped silica nano-pores: direct comparison with quasielastic neutron scattering experiments. Phys. Chem. Chem. Phys., 2017, 19, 32320-32332. 
411 [24] Dhiman, I., Shrestha, U. R., Bhowmik, D., Cole, D. R., and Gautam, S. (2019). Influence of 412 molecular shape on self-diffusion under severe confinement: A molecular dynamics study.

413 Chem. Phys., 2019, 516, 92-102.

414 [25] Dhiman, I., Bhowmik, D., Shrestha, U. R., Cole, D. R., \& Gautam, S. Effect of molecular 415 shape on rotation under severe confinement. Chem. Eng. Sc., 2018, 180, 33-41.

416 [26] Gautam, S.; Kolesnikov, A. I.; Rother, G.; Dai, S.; Qiao, Z. A.; Cole, D. Effects of 417 Confinement and Pressure on the Vibrational Behavior of Nano-Confined Propane. J. Phys. 418 Chem. A 2018, 122, 6736-6745.

419 [27] Chathoth, S. M.; He, L.; Mamontov, E.; Melnichenko, Y. B. Effect of Carbon Dioxide and 420 Nitrogen on the Diffusivity of Methane Confined in Nano-Porous Carbon Aerogel. Microporous 421 Mesoporous Mater. 2012, 148, 101-106.

422 [28] Salles, F.; Jobic, H.; Devic, T.; Guillerm, V.; Serre, C.; Koza, M. M.; Ferey, G.; Maurin, G. 423 Diffusion of Binary $\mathrm{CO}_{2} / \mathrm{CH}_{4}$ Mixtures in the MIL-47(V) and MIL-53(Cr) Metal - Organic 424 Framework Type Solids: A Combination of Neutron Scattering Measurements and Molecular 425 Dynamics Simulations. J. Phys. Chem. C 2013, 117, 11275-11284.

426 [29] Le, T.; Ogbe, S.; Striolo, A.; Cole, D. R. N-octane diffusivity enhancement via carbon 427 dioxide in silica slit-shaped nanopores - a molecular dynamics simulation. Mol. Simul. 2016, 42, $428745-752$.

429 [30] Le, T., Striolo, A., Cole, D. R. $\mathrm{CO}_{2}-\mathrm{C}_{4} \mathrm{H}_{10}$ mixtures simulated in silica slit pores: relation 430 between structure and dynamics. J. Phys. Chem. C, 2015, 119(27), 15274-15284.

431 [31] Chakraborty, S., Kumar, H., Dasgupta, C. Maiti, P. K. Confined water: Structure, 432 dynamics, and thermodynamics. Acc. Chem. Res., 2017, 50, 2139-2146.

433 [32] Cerveny, S., Mallamace, F., Swenson, J., Vogel, M., Xu, L. Confined water as model of 434 supercooled water. Chem. Rev., 2016, 116, 7608-7625.

435 [33] Phan, A., Cole, D. R., Weiß, R. G., Dzubiella, J., Striolo A. Confined water determines 436 transport properties of guest molecules in narrow pores. ACS Nano. 2016, 10, 7646 - 7656. 
437 [34] Bui T., Phan A., Cole D. R., Striolo A. Transport Mechanism of Guest Methane in Water438 Filled Nano-Pores. J. Phys. Chem. C 2017, 121, 15675 - 15686.

439 [35] Le, T. T. B., Striolo, A., Gautam, S. S., Cole, D. R. Propane-water mixtures confined within 440 cylindrical silica nanopores: structural and dynamical properties probed by molecular dynamics. 441 Langmuir, 2017, 33, 11310-11320.

442 [36] Mamontov, E.; Herwig, K. W. A Time-of-Flight Backscattering Spectrometer at the 443 Spallation Neutron Source, BASIS. Rev. Sci. Instrum. 2011, 82, 085109.

444 [37] Azuah, R. T.; Kneller, L. R.; Qiu, Y.; Tregenna-Piggott, P. L. W.; Brown, C.M.; Copley, J. 445 R. D. Dimeo,R.M. DAVE: A Comprehensive Software Suite for the Reduction, Visualization, 446 and Analysis of Low Energy Neutron Spectroscopic Data. J. Res. Natl. Inst. Stand. Technol. $447 \quad 2009,114,341-358$.

448 [38] Demiralp, E.; Cagin, T.; Goddard, W. A. Morse stretch potential charge equilibrium force 449 field for ceramics: Application to the quartzstishovite phase transition and to silica glass. Phys. 450 Rev. Lett. 1999, 82 (8), 1708-1711.

451 [39] Cygan, R. T.; Liang, J. J.; Kalinichev, A. G. Molecular models of hydroxide, oxyhydroxide, 452 and clay phases and the development of a general force field. J. Phys. Chem. B 2004, 108 (4), $453 \quad 1255-1266$.

454 [40] Abascal, J. L. F., Sanz, E., García Fernández, R., \& Vega, C. A potential model for the 455 study of ices and amorphous water: TIP4P/Ice. J. Chem. Phys. 2005,122(23), 234511.

456 [41] Martin, M. G.; Siepmann, J. I. Transferable potentials for phase equilibria. 1. United-atom 457 description of n-alkanes. J. Phys. Chem. B 1998, 102 (14), 2569-2577.

458 [42] Essmann, U.; Perera, L.; Berkowitz, M. L.; Darden, T.; Lee, H.; Pedersen, L. G. A Smooth 459 Particle Mesh Ewald Method. J. Chem. Phys. 1995, 103 (19), 8577-8593.

460 [43] Allen, M. P.; Tildesley, D. J. Computer Simulation of Liquids Oxford University Press: 461 Oxford, U.K., 2004.

462 [44] M. Bee, Quasielastic Neutron Scattering, Adam Hilger, Bristol, 1988. 
463 [45] Singwi, K. S.; Sjölander, A. Diffusive Motions in Water and Cold Neutron Scattering. Phys. 464 Rev. 1960, 119, 863-871

465 [46] Greiner-Schmid, A.; Wappmann, S.; Has, M.; Lüdemann, H. D.Self-Diffusion in the 466 Compressed Fluid Lower Alkanes: Methane,Ethane, and Propane. J. Chem. Phys. 1991, 94, $467 \quad 5643-5649$.

468 [47] Cole, D. R. and Striolo, A. (2019) Chapter 12. The Influence of nanoporosity on the 469 behavior of carbon-bearing fluids. In: Deep Carbon: Past and Present. (editors - B. Orcutt, I. 470 Daniel, R. Dasgupta), Cambridge University Press, 358-387.

471

472 [48] Ho, T. A., Striolo, A. Water and methane in shale rocks: Flow pattern effects on fluid 473 transport and pore structure. AIChE Journal, 2015, 61(9), 2993-2999.

474 